\title{
Druck im Job - Dauerdruck auf die Gefäße
}

\author{
Chefredakteur Dr. med. Dirk Einecke
}

\section{HOCHDRUCKTHERAPIE}

\section{Wissenschaftliche Leitlinien oder politische Richtlinien?}

Die Hochdruckliga tagt, und das IOWIG arbeitet gerade an einer Bewertung unterschiedlicher Antihypertensiva - ein idealer Anlass für eine Podiumsdiskussion zum Auftakt der Ligatagung in München. Laut Tagungspräsident Prof. Martin Middeke besteht bei der wissenschaftlichen Gesellschaft die Sorge, dass die patentgeschützten Sartane als einzige Antihypertensiva ohne spezifische Nebenwirkungen aufgrund des höheren Preises als wenig wirtschaftlich beurteilt werden. Hochdruckpatienten haben keine Beschwerden, da spielen Lebensqualität, Nebenwirkungen und Compliance eine entscheidende Rolle in der Verhinderung von Organkomplikationen, betont LigaVorstand Prof. Helmut Haller. Seine Botschaft: Wenn diese Parameter bei der Begutachtung nicht berücksichtigt werden, ist weder dem Patienten noch der Solidargemeinschaft geholfen.

Prof. P. Sawicki, Leiter des IOWIG, möchte die Diskussion nicht nur auf die Medikamente fokussieren. Wir haben genügend Medikamente zur Verfügung, und wenn es Unterschiede gibt, so sind diese vermutlich gering - so sein Standpunkt.
Wenn bei uns nur ein Viertel aller Hypertoniker gut behandelt ist, dann liegen andere Gründe vor. Aufklärung, Prävention, Allgemeinmaßnahmen und die Umorganisation der ärztlichen Tätigkeit weg von der 7-Minuten-Sprechstunde sind weitaus dringlichere Probleme, so Sawicki.

\section{HYPERTONIE}

\section{Risikofaktor beruflicher Stress}

Ein aufreibender 10-Stunden-Arbeitstag, Ärger mit dem Chef und Unverständnis im privaten Umfeld: Das treibt den Blutdruck in die Höhe, und zwar dauerhaft, wie aktuelle Daten der STARLET-Studie zeigen. Für die deutsche Studie war bei fast 3500 Berufstätigen über einen Zeitraum von fünf Jahren mehrmals eine ambulante 24-Stunden-RR-Messung durchgeführt worden. Außerdem mussten die Teilnehmer die psychische Belastung durch ihre Arbeit bewerten. Das Ergebnis: Hypertoniker klagten öfter über Negativstress am Arbeitsplatz. Nahm der Stress über die Jahre zu, stieg nicht nur das Risiko für höhere RR-Werte, sondern auch die Rate an kardiovaskulären Ereignissen. Wenn der Druck im Job nachließ, sanken Blutdruck und Herz-Kreislauf-Risiko.

Lüders S et al.; Hochdruckliga-Tagung, München, 22. November 2006

\section{AU-Zeiten auf „historischem Tief“}

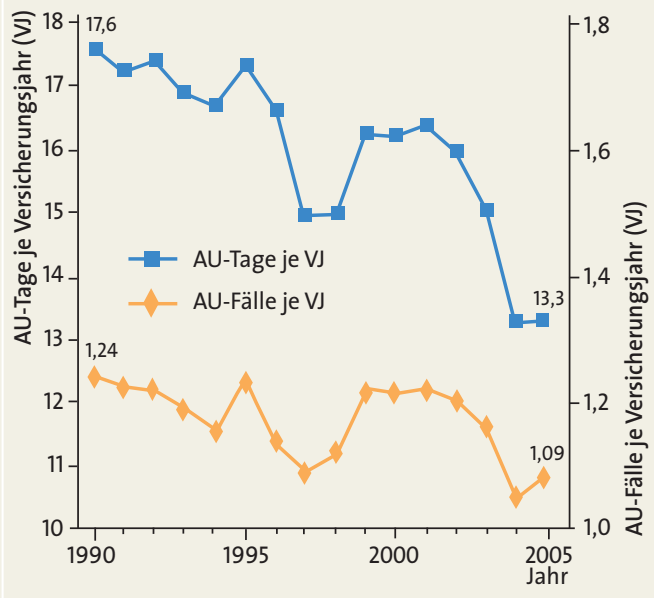

\section{GEK-REPORT}

\section{Ärzte schreiben weniger krank}

Die Zahl der krankheitsbedingten Fehltage hat in Deutschland einen „historischen Tiefstand“ erreicht, wie der aktuelle GEKReport feststellt. In den Jahren 2004 und 2005 waren Erwerbstätige durchschnittlich an 13,3 Tagen krankgeschrieben - im Jahr 1995 waren es 17,3 Tage. Mit rund ein Drittel der Fälle die häufigste Ursache für AU-Meldungen sind Atemwegserkrankungen. Die längsten Ausfallzeiten verursachen Erkrankungen des Bewegungsapparats.

\section{DURCH REGELMÄSSIGE BEWEGUNC}

\section{COPD-Komplikationen entkommen}

Regelmäßige körperliche Aktivität scheint bei Patienten mit chronisch obstruktiver Lungenerkrankung (COPD) den Krankheitsverlauf und die Prognose günstig zu beeinflussen. In einer dänischen Kohortenstudie hatten Patienten, die mindestens zwei Stunden pro Woche spazieren gingen oder Rad fuhren, ein um $30-40 \%$ geringeres Risiko für COPD-bedingte Krankenhauseinweisungen und Mortalität. Dieser Zusammenhang bestand unabhängig vom Schweregrad der Erkrankung. Die Studienautoren schlagen deswegen vor, in COPD-Leitlinien die Empfehlung zu körperlicher Aktivität mitaufzunehmen. Thorax 2006;61:772-8

\section{PLÄTTCHENAGGREGATIONSHEMMER}

\section{Schokolade statt ASS?}

Nicht nur ASS kann die Blutgerinnung hemmen. Schokolade hat offensichtlich vergleichbare Eigenschaften, fanden Forscher der John-Hopkins-Universität in Baltimore heraus. Schon zwei Teelöffel pro Tag zeigen einen Effekt. Allerdings muss es die dunkle, bittere Schokolade sein, die hellen Sorten enthalten zu viel Zucker und Fett.

Jahrestagung der American Heart Association, Chicago, 11.-15.11.2006

\section{TREUE IN DER PARTNERSCHAFT}

\section{Immunsystem will Abwechslung}

Für notorische Fremdgeher gibt es jetzt möglicherweise eine gute Ausrede: „Liebling, unser Immunsystem ist zu ähnlich.“ Jedenfalls fanden Forscher in New Mexico heraus, dass Frauen, deren Immunsystem große Ähnlichkeit zu dem ihres Partners aufweist, am ehesten zu Seitensprüngen neigen. Bei Männern war ein solcher Zusammenhang nicht feststellbar - sie müssen sich weiterhin andere Entschuldigungen ausdenken.

Psychological Science 2006;17:830 